\title{
Tumor Microenvironment Targeting and Responsive Peptide-Based Nanoformulations for Improved Tumor Therapy
}

\author{
Hao Qin, Yanping Ding, Ayeesha Mujeeb, Ying Zhao, and Guangjun Nie \\ CAS Key Laboratory for Biomedical Effects of Nanomaterials and Nanosafety and CAS Center for Excellence in Nanoscience, \\ National Center for Nanoscience and Technology (H.Q., Y D., A.M., Y. Z., G.N.), and University of Chinese Academy of Sciences \\ (H.Q., Y.D., Y.Z., G.N.), Beijing, China
}

Received January 3, 2017; accepted April 12, 2017

\section{ABSTRACT}

The tumor microenvironment participates in all stages of tumor progression and has emerged as a promising therapeutic target for cancer therapy. Rapid progress in the field of molecular selfassembly using various biologic molecules has resulted in the fabrication of nanoformulations that specifically target and regulate microenvironment components to inhibit tumor growth. This inhibition process is based on differentiating between biophysicochemical cues guiding tumor and normal tissue microenvironments. Peptides and peptide derivatives, owing to their biocompatibility, chemical versatility, bioactivity, environmental sensitivity, and biologic recognition abilities, have been widely used as building blocks to construct multifunctional nanostructures for targeted drug delivery and controlled release. Several groups of peptides have been identified as having the ability to penetrate plasma membranes, regulate the essential signaling pathways of angiogenesis and immune reactions, and recognize key components in the tumor microenvironment (such as vascular systems, stromal cells, and abnormal tumor biophysicochemical features). Thus, using different modules, various functional peptides, and their derivatives can be integrated into nanoformulations specifically targeting the tumor microenvironment with increased selectivity, on-demand response, elevated cellular uptake, and improved tumor therapy. In this review, we introduce several groups of functional peptides and highlight peptide-based nanoformulations that specifically target the tumor microenvironment. We also provide our perspective on the development of smart drug-delivery systems with enhanced therapeutic efficacy.

\section{Introduction}

Tumors are composed of tumor cells and their microenvironment, including various stromal cells, extracellular matrix (ECM), soluble cytokines, and tumor vascular systems. The interaction between tumor cells and the surrounding microenvironment occurs at all stages of tumor progression. Hypoxia results in tumor vascular endothelial cells being activated, with aberrant expression of cell surface and secreted proteins, including integrins and matrix metalloproteinases (MMPs), that facilitate the construction of malformed and dysfunctional new

The writing of this review was supported by the grants from the Ministry of Science and Technology of the People's Republic of China [Grant 2013CB932701], National Natural Science Foundation of China [Grant 21373067, 51673051, 31325010, and 11621505], Beijing Municipal Science \& Technology Commission [Grant Z161100000116035], Youth Innovation Promotion Association of Chinese Academy of Science [Grant 2017056], and the Frontier Research Program of Chinese Academy of Science [Grant QYZDJSSW-SLH022].

https://doi.org/10.1124/mol.116.108084. blood vessels (Carmeliet, 2000). Cancer-associated fibroblasts (CAFs), the most abundant cell type in the tumor microenvironment, actively participate in ECM remodeling and in promoting tumor growth (Neri et al., 2016). Tumor-associated macrophages (TAMs) are the predominant inflammatory cells in malignant solid tumors. Most of the TAMs polarize toward M2like macrophages, which are characterized as immunosuppressive and play an important role in angiogenesis (Rogers and Holen, 2011). In addition, all stromal cells and ECM in the tumor microenvironment can form physiologic barriers, sheltering tumor cells and contributing to drug resistance (Khawar et al., 2015).

Improved therapeutic outcomes can potentially be achieved by synergistically targeting tumor cells and regulating the tumor microenvironment. Compared with the normal tissue environment, the tumor microenvironment exhibits stromal cell abnormalities, aberrant protein expression, acidosis, and hypoxia. These differences provide multiple targets for the selective delivery of therapeutic agents to tumors (Ji et al., 2013).

ABBREVIATIONS: Ang, angiopoietin; CAF, cancer-associated fibroblast; CPP, cell-penetrating peptide; CTL, cytotoxic T lymphocyte; DEAP, 3-diethylaminopropylisothiocyanate; Dox, doxorubicin; ECM, extracellular matrix; FAP- $\alpha$, fibroblast activation protein- $\alpha$; FGF-12, fibroblast growth factor-12; GEM, gemcitabine; K-FGF, Kaposi fibroblast growth factor; LCST, low critical solution temperature; MMP, matrix metalloproteinase; PD-1, programmed cell death protein 1; PD-L1, programmed death-ligand 1; PEG, poly(ethylene glycol); TAM, tumor-associated macrophage; VEGF, vascular endothelial growth factor; VEGFR2, VEGF receptor 2. 
The emergence and progression of nanotechnology provide a novel toolbox for designing the next generation of intelligent therapeutics with the design of well defined structures with flexible composition. By carefully selecting the building blocks and modulating the physicochemical properties of different nanoformulations, elevated tumor accumulation, on-demand drug release, and enhanced therapeutic outcomes can be achieved. With deeper understanding of the features of key components within the tumor microenvironment and the mechanisms by which these components promote tumor progression, therapeutic nanoformulations have been designed to specifically target and regulate the tumor microenvironment (Danhier et al., 2010). In addition, the physicochemical differences between normal tissues and the tumor microenvironment, such as acidosis and hypoxia, have also been taken advantage of to construct well controlled nanosystems with high therapeutic efficacy (Mura et al., 2013).

Endowed with low immunogenicity, facile modification, ample bioactivity, and the ability to penetrate tissues, peptides have served as promising building blocks for the construction of advanced nanoformulations. Many peptides used in tumor therapy are derived from functional domains of proteins and exhibit specific bioactivities, such as receptor binding, structural sensitivity to changing local physiologic or chemical conditions, penetration of the plasma membrane, and activation or inhibition of cellular pathways. Several research groups are screening more efficient functional peptides (Laakkonen et al., 2002; Porkka et al., 2002; Cieslewicz et al., 2013; Gautam et al., 2014). Owing to their salient bioactive functions, peptides hold great potential to become one of the most extensively used building blocks for the construction of nanoformulations for cancer treatment. Peptides can be incorporated into modules of nanoformulations that include targeting ligands, responsive cleavage sites, internalization mediators, and therapeutic molecules. In addition, taking advantage of the solid-phase peptide synthesis method, a sophisticated technique with high synthetic efficiency and moderate reaction conditions, one can easily obtain desired peptides with defined functions. In this review, we describe several groups of functional peptides with distinct bioactive properties and discuss progress focused on the design and fabrication of peptide-based nanoformulations specifically targeting the tumor microenvironment.

\section{Functional Peptides}

Tumor Microenvironment Targeting Peptides. Functionalization with tumor-targeting motifs is an excellent strategy for increasing the accumulation of therapeutic agents within tumor tissues. With advancements in screening techniques, such as the phage-displayed peptide library and the one-bead one-compound combinatorial library, peptides have been identified that possess tumor-homing capacity. These peptides can specifically bind to various receptors that are specifically expressed or overexpressed in tumor tissues. Compared with antibodies, targeting peptides are much smaller, which makes them more efficient in tissue penetration and relatively easy to fabricate at much lower cost. In comparison with other small molecular targeting agents, peptides may be more straightforward for molecular design and more biocompatible. A brief summary of some targeting peptides is provided in Table 1.

The tumor vasculature is the first checkpoint that therapeutic systems encounter when circulating into the tumor. This makes vascular constituents, including endothelial cells, pericytes, and blood components, attractive targets. RGD and NGR, the first two targeting peptides screened via phagedisplayed peptide libraries (Pasqualini et al., 1997; Arap et al., 1998), are widely used in targeted delivery of therapeutic agents to tumor tissues. These short tripeptides bind to integrin $\alpha_{\mathrm{V}} \beta_{3}$ and $\alpha_{\mathrm{V}} \beta_{5}$ and aminopeptidase $\mathrm{N}$, respectively, which are overexpressed on vascular endothelial cells of tumor angiogenic blood vessels, resulting in remarkable accumulation of cargoes within the tumor. Other than RGD and NGR, functional peptides such as IF7, F3, cytotoxic T-lymphocyte (CTL), and CREAK also exhibit the ability to specifically target tumor blood vessels. The receptor for IF7 targeting is annexin1, a highly specific surface marker of tumor vasculature (Hatakeyama et al., 2011). F3, which is derived from the $\mathrm{N}$-terminal fragment of human high-mobility group protein 2 , possesses high affinity for nucleolin proteins expressed on the surface of tumor cells and endothelial cells (Porkka et al., 2002). The fibrin-fibronectin complex of plasma clots in tumor vessel walls and interstitial spaces, formed as a result of leaky blood vessels, provides another well recognized target for tumor targeting. The cyclic nonapeptides CLT1 and CLT2 strongly accumulate in tumor blood vessels and stroma via their interaction with fibrin-fibronectin (Pilch et al., 2006). Another peptide, CREAK, binds to clotted plasma proteins and induces additional local clotting, thereby producing new targets for additional peptide binding (Simberg et al., 2007). This selfamplifying process is an excellent feature for enhanced targeting efficiency.

Tumor stromal cells with unusual protein expression are also good candidates for targeting. Lyp-1, a cyclic nonapeptide,

TABLE 1

Brief summary of targeting peptides

\begin{tabular}{lllll}
\hline Targeting Position & Names & & Sequence & Receptors \\
\hline Tumor vasculature & RGD & RGD & Integrin $\alpha_{\mathrm{V}} \beta_{3} \alpha_{\mathrm{V}} \beta_{5}$ & Pasqualini et al., 1997 \\
& NGD & NGD & Aminopeptidase N & Arap et al., 1998 \\
& IF7 & IFLLWQR & Annexin 1 & Hatakeyama et al., 2011 \\
& F3 & KDEPQRRSARLSAKPAPPKPEPKPKKAPAKK & Nucleolin & Porkka et al., 2002 \\
& CTL1 & CGLIIQKNEC & Clotted plasma protein & Pilch et al., 2006 \\
& CTL2 & CNAGESSKNC & Clotted plasma protein & Pilch et al., 2006 \\
TAM & CREAK & CREAK & Clotted plasma protein & Simberg et al., 2007 \\
& Lyp-1 & CGNKRTRGC & P32/gC1q & Uchida et al., 2011 \\
MSC & M2pep & YEQDPWGVKWWY & Unclear & Cieslewicz et al., 2013 \\
ECM & WAT & CSWKYWFGEC & Unclear & Daquinag et al., 2011 \\
& $a$ & CRRHWGFEF & MMP-2/9 & Koivunen et al., 1999 \\
& $a$ & CTTHWGFTLC & MMP-2/9 & Koivunen et al., 1999 \\
\hline
\end{tabular}

MSC, mesenchymal stromal cell.

${ }^{a}$ No specific names. 
has been verified to selectively bind to $\mathrm{p} 32 / \mathrm{gC} 1 \mathrm{q}$, a receptor abundant on the surface of TAMs, as well as on tumor lymphatics and tumor cells (Laakkonen et al., 2002; Fogal et al., 2008; Uchida et al., 2011). When expressed in normal tissues, p32 exists intracellularly as a mitochondrial protein and escapes peptide recognition. One group identified a unique M2-selective peptide, called M2pep, that specifically recognizes murine M2 cells, including TAMs, and has low affinity for other leukocytes (Cieslewicz et al., 2013). Another targeting molecule, WAT, is a cyclic peptide that homes to adipose stromal cells (Daquinag et al., 2011), which belong to the mesenchymal stromal cell lineage.

In addition to cytokines and enzymes, ECM presents other appealing therapeutic targets. MMPs, overexpressed by endothelial cells and tumor cells, play an important role in tumor growth, angiogenesis, and metastasis. Two peptides, CTTHWGFTLC and CRRHWGFEFC, have been shown to selectively target MMP-2 and MMP-9 (Koivunen et al., 1999). The two peptides display high affinity and also inhibit enzymatic activities of MMP-2 and MMP-9 (Koivunen et al., 1999). Specific targeting with simultaneous inhibition suggests the potential for enhanced targeting and antitumor efficacy.

Microenvironment Responsive Peptides. Since proteinases and peptidases are abundant in the tumor interstitial space, therapeutic nanosystems fused with peptides that are specific substrates of these enzymes can be designed to control the release of therapeutic agents within the tumor microenvironment. As described already herein, MMPs, with an elevated expression in tumor ECM, are crucial in tumor progression. Therefore, making use of MMP-cleavable sequences can readily achieve drug release or active site exposure. The substrate peptide for MMP-2 is GPLGIAGQ; this sequence is cleaved into GPLG and IAGO by MMP-2. One group developed a liposome modified with cell-penetrating peptide (CPP) and a poly(ethylene glycol) (PEG)-conjugated antibody (Gao et al., 2013). PEG was linked to the liposome via a MMP-2 responsive sequence. In the presence of MMP-2, long PEG chains were removed from the liposomes. As a result, the exposed CPP mediated the internalization of the liposomes. In another example, the MMP-2 and MMP-9 sensitive sequence, PVGLIG, can be cleaved between glycine and leucine (Gao et al., 2013). This sequence can also be used for construction of tumor niche-responsive nanoformulations. fibroblast activation protein- $\alpha$ (FAP- $\alpha$ ) is another accessible protease that is specifically expressed on the surface of CAFs, a major cellular component in the tumor microenvironment. FAP- $\alpha$ selectively cleaves the sequence GPAX (X designates any amino acid) between proline, and alanine (Ji et al., 2016b). Microenvironment responsive peptides are listed in Table 2.

The sustained Warburg effect and limited clearance of metabolic acids leads to a more acidic $\mathrm{pH}$ within the tumor microenvironment than in normal tissues. The relative acidity has an important implication on specific responses of the tumor microenvironment. pHLIP is a peptide that has striking features of low $\mathrm{pH}$ sensitivity and tumor targeting (Andreev et al., 2007). This peptide, which is part of the bacteriorhodopsin $\mathrm{C}$ helix, inserts across the membrane as an $\alpha$-helix at low $\mathrm{pH}$. In a basic or neutral environment, the peptide is largely unstructured and has low affinity for cell membranes. Polyhistidines also possess the ability to respond to the acidic tumor environment; this is because an imidazole group on

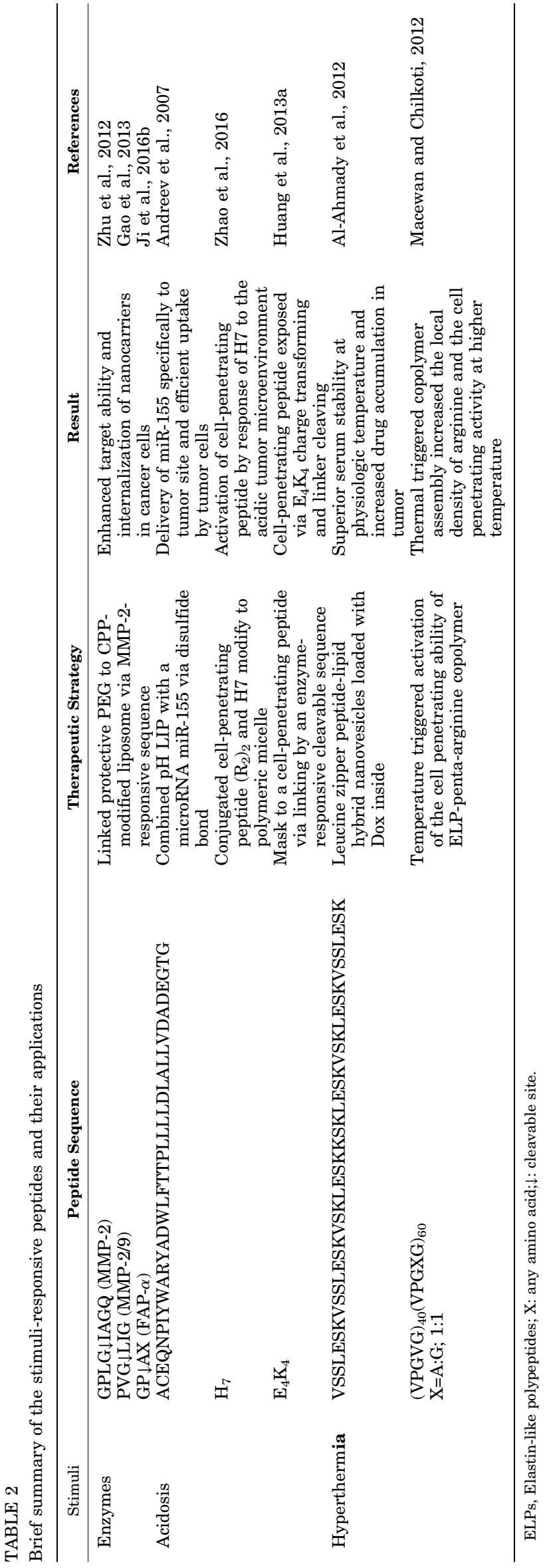


histidine, with a pKa of approximately 6.5, can be protonated under acidic conditions to make the peptide more hydrophilic (Zhao et al., 2016). The disturbed polarity usually leads to structural transformation of nanoformulations to exert favorable effects such as drug release and functional site exposure. Another group of pH-responsive peptides include sequences of carefully designed acidic and basic amino acids with isoelectric points between 6 and 7. One of the peptides includes an $\mathrm{E}_{4} \mathrm{~K}_{4}$ sequence, which has a $\mathrm{pI}$ of approximately 6.4 and is negatively charged under physiologic conditions; in an acidic tumor microenvironment, this peptide is uncharged or slightly positively charged (Huang et al., 2013a). The changes in hydrophilicity or net charge can be used to alter conformations supported by electrostatic attractions to induce functional domain exposure or cargo release. These microenvironment responsive peptides are described in Table 2.

Mild hyperthermia is another feature of tumor tissue that occurs as a result of dysfunctional tumor vascular systems and poor heat exchange. Slightly higher temperatures, compared with the surrounding tissues, can potentially trigger particular peptides to respond to the tumor microenvironment. A leucine zipper peptide has been shown to form coiled-coil selfassembled aggregates composed of $\alpha$-helix monomers; these dissociate into disordered, unstructured monomers at temperatures higher than $40^{\circ} \mathrm{C}$. In this transformation, the peptide loses the ordered original structure, facilitating the release of drugs (Al-Ahmady et al., 2012). Elastin-like polypeptides are another group of temperature-responsive peptides that switch conformations in response to different temperature conditions (Macewan and Chilkoti, 2012). They possess low critical solution temperature (LCST), exhibit properties as soluble unimolecules below their LCST, and assemble into aggregates when the temperature is greater than the LCST. Amphiphilic Elastin-like polypetides can also self-assemble into micelles with a hydrophobic core and hydrophilic corona (Macewan and Chilkoti, 2012).

Tissue-/Cell-Penetrating Peptides. Physiologic barriers such as vascular endothelial cells, tumor stromal cells, and ECM in tumor tissues are known to hinder the efficient penetration of nanosystems to reach targeted cells. The selective permeability of the plasma membrane leads to insufficient internalization of therapeutic systems. One type of tissue-penetrating motif, called the CendR motif, with a sequence $\mathrm{R} / \mathrm{KXXR} / \mathrm{K}$ and a second arginine or lysine residue on the $\mathrm{C}$ terminus of the peptide, has shown to bind to neuropilin-1, a membrane receptor expressed on endothelial cells, and to activate cell internalization and trans-tissue transportation (Wang et al., 2011). iRGD (CRGDKGPD) (Sugahara et al., 2010), one of the sequences within the CendR motif, exhibits tumor-specific penetrating ability as a result of the tumor-homing RGD motif. The RGD sequence mediates binding of iRGD to the tumor vascular targets, integrin $\alpha_{\nu} \beta_{3}$ and $\alpha_{\nu} \beta_{5}$. Subsequent proteolysis generates the $\mathrm{C}$-terminal $\mathrm{R} / \mathrm{KXXR} / \mathrm{K}$ motif, which activates the neuropilin-1-dependent penetrating pathway. Another type of CPP, which is varied in size and sequence, interacts with the lipid bilayer and internalizes into cells via clathrin- or caveolin-mediated endocytosis, micropinocytosis, or an endocytosis-independent mechanism, such as the carpet model, inverted micelle model, barrel stave pore model, or toroidal model (Trabulo et al., 2010).
Most CPPs are derived from segments of natural translocating proteins with a large variety of sizes, sequences, and physiochemical properties. They can be classified into three main categories: cationic, hydrophobic, and amphipathic peptides. Cationic CPPs are positively charged and have a high affinity to negatively charged plasma membranes. The most commonly used cationic CPPs are poly-arginine and Tatderived peptides (Takeshima et al., 2003; Walrant et al., 2011). Hydrophobic CPPs comprise nonpolar amino acids with low net charge and high lipid affinity. Signal sequence Kaposi fibroblast growth factor (K-FGF) and fibroblast growth factor12 (FGF-12) belongs to the hydrophobic CPP category (Dokka et al., 1997; Nakayama et al., 2011). The remaining CPPs belong to amphipathic CPP class, which includes Antp, pVEC, penetratin, transportan 10, M918, VP22, and SAP (Derossi et al., 1994; Elliott and O'Hare, 1997; Fernandez-Carneado et al., 2004; Elmquist et al., 2006; El-Andaloussi et al., 2007; Amand et al., 2008; Islam et al., 2014). Amphipathic CPPs contain both polar and nonpolar amino acids and are thus both hydrophobic and hydrophilic in nature. There is a special group of amphipathic CPPs, including MPG, Pep-1, and $\mathrm{S}_{13^{-}}$ PV, that are synthesized by fusing segments of the HIV GP41 protein, reverse transcriptase of human immunodeficiency virus type 1, or DermaSeptin (Dermarite Industries, LLC, North Bergen, NJ) S4 peptide with the nuclear localization signal of simian virus 40 large $\mathrm{T}$ antigen (Hariton-Gazal et al., 2002; Morris et al., 2008). The resulting peptides form stable complexes with their cargo via noncovalent interactions and penetrate into the cell with high efficiency. A summary of the most widely used CPPs is presented in Table 3.

Therapeutic Peptides. Peptides have received considerable attention as therapeutic agents because of their high specificity, low toxicity, good tissue penetration, cost-effectiveness, and easy modification. Therapeutic peptides are commonly derived from chemokines, ECM proteins, growth factors, antigens, and other proteins, or they are identified via screening of a phage-displayed peptide library. They selectively target particular receptors to either activate essential signaling pathways or inhibit the receptors from interacting with their ligands. A great number of tumor therapeutic peptides have been identified, especially in the fields of antiangiogenesis and immunotherapy. Some examples are summarized in Table 4.

Sustained formation of new blood vessels is an important hallmark of tumor progression. Inhibiting angiogenesis and normalizing tumor vascular systems are the strategies most emphasized in therapy targeted to the tumor vasculature. Many peptides have been reported to target tumor blood vessels and block angiogenesis; these include T4, C16Y, and 6a-P (Rosca et al., 2011). T4 is a peptide identified by screening of a phage-displayed peptide library for high affinity to Tie2, an endothelial cell receptor kinase important in angiogenesis (Tournaire et al., 2004). Binding of T4 to Tie2 not only inhibits the interactions between Tie2 and angiopoietin 2 (Ang2) or angiopoietin 1 (Ang1) but also suppresses angiopoietin 1-dependent ERK activation and migration in human umbilical vascular endothelial cells. $\mathrm{C} 16 \mathrm{Y}$ is an antiangiogenic peptide derived from one of the most potent angiogenic sites, C16 in laminin-1. This peptide eliminates the attachment of endothelial cells to laminin-1, disrupts tube formation, and inhibits angiogenesis in the tumor site (Ponce et al., 2003). $6 \mathrm{a}-\mathrm{P}$ is a 20 amino acid peptide derived from vascular 


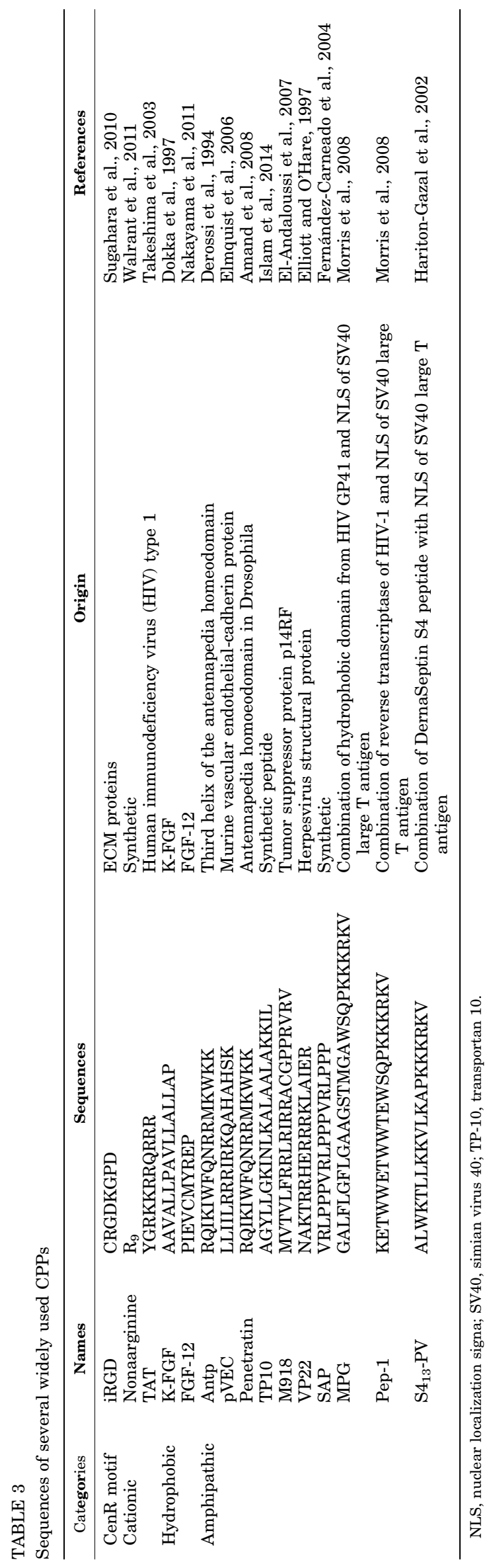

endothelial growth factor (VEGF). It binds to heparin sulfate proteoglycan, which is an important regulator in angiogenesis; the binding consequently inhibits the interaction between VEGF and heparin sulfate proteoglycan (Lee et al., 2010).

Because of its potential for remarkable specificity and efficacy, tumor immunotherapy has emerged as a promising tumor therapeutic strategy. Peptides play a significant role in tumor immunotherapy. Examples include peptide vaccines and immune checkpoint blocking peptides. Tumor antigens that can be recognized by cytotoxic T lymphocytes (CTLs) are usually expressed to a limited extent on tumor cells; however, peptides derived from tumor-associated antigens possess great potential to be recognized by CTL and can be delivered to tumor cells. For example, peptides derived from glypican-3 have efficiently stimulated CTL activity when loaded into tumors in vivo via intratumoral injection (Nobuoka et al., 2013). One of the mechanisms that allow tumor cells to escape from the immune system is overexpression of immune checkpoint proteins to inhibit T-cell attack. Using exogenous antagonists to block immune checkpoints is a promising strategy to disturb immune-suppressing pathways and activate the antitumor immune response of T cells. ${ }^{\mathrm{D} P P A}-1$, a D-peptide antagonist, targets programmed death-ligand 1 (PD-L1) and efficiently disrupts the interaction between PD-L1 and its receptor, programmed death 1 (PD-1), unleashing the antitumor immune reaction (Chang et al., 2015). ${ }^{\mathrm{D} P P A}-1$ is the first reported proteolysis-resistant peptide antagonist targeting the immune checkpoint protein human PD-1/PD-L1 (Chang et al., 2015).

Peptide toxins can also be used to regulate the tumor microenvironment by reducing the number of stromal cells to deteriorate the tumor-supporting stroma and break physiologic barriers for better drug perfusion. Numerous peptide toxins have been used to kill tumor cells, such as proapoptotic peptide KLA, phalloidin, and amanitin (An et al., 2010; Moshnikova et al., 2013; Jung et al., 2016), and they can also be used to induce stromal cell toxicity for enhanced drug perfusion and antitumor therapeutic efficacy.

\section{Tumor Microenvironment Targeting and Responsive Peptide-Based Nanoformulations}

As already described herein, peptides have exhibited various activities in the tumor microenvironment. For example, targeting peptides show high affinity to components of the tumor microenvironment, and responsive peptides are sensitive to tumor physicochemical conditions, CPPs are able to enhance cellular uptake, and therapeutic peptides can regulate pathways related to tumor progression. A number of these attractive building blocks have been used to construct nanoformulations specifically targeting tumor microenvironment and have achieved exciting treatment outcomes. In this section, we summarize recent work published by our group and others on the use of peptide-based nanoformulation strategies, especially for regulating tumor blood vessels and stromal cells.

\section{Ligand Peptides Mediate Nanoformulations to Target Tumor Microenvironment}

Herein, we review nanoformulations that are conjugated with targeting peptides against tumor vasculature, 


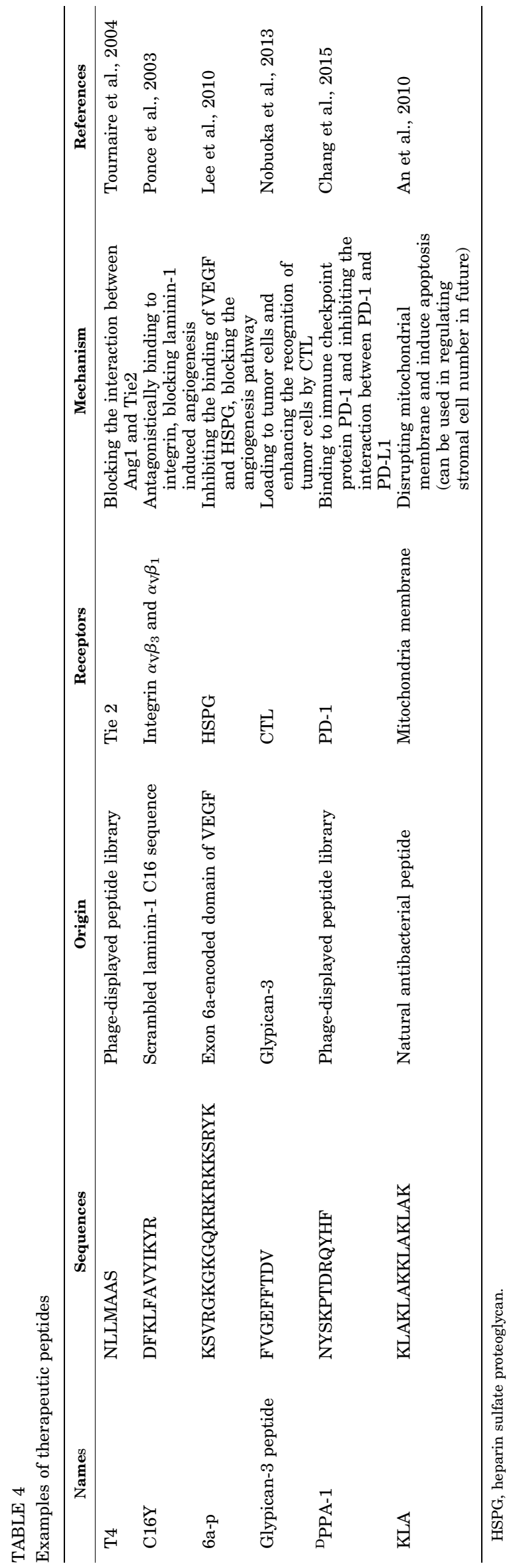

lymphatics, and stromal cells and carry cargo, such as chemotherapy or gene therapy agents, for improved tumor therapy and decreased systemic toxicity.

Targeting Tumor Vasculatures. The most studied peptides targeting tumor blood vessels are the derivatives of RGD, an integrin $\alpha_{\mathrm{V}} \beta_{3}$ and $\alpha_{\mathrm{V}} \beta_{5}$ binding sequence present in various ECM proteins, including fibronectin and vitronectin. Some studies have suggested that modification of RGD ligands for use in nanoformulations carrying chemotherapeutic agents, siRNA, or small-molecule inhibitors leads to enhanced inhibition of angiogenesis and tumor growth (Danhier et al., 2012). Interestingly, an antimetastatic effect was observed was observed when integrin $\alpha_{\nu} \beta_{3}$ and $\alpha_{\nu} \beta_{5}$ were targeted alone (Gvozdenovic et al., 2016); further studies are needed to confirm this promising strategy for inhibition of tumor metastasis.

VEGF, also known as vascular permeability factor, plays a vital role in the angiogenic process by binding to specific VEGF receptor 2 (VEGFR2, also known as KDR/Flk-1), a tyrosine kinase receptor. The binding of VEGF and its receptor then activates downstream signaling pathways, including FAK/paxillin and RAS/ERK, and results in the proliferation and migration of endothelial cells, consequently promoting angiogenesis and vascular growth (Liang et al., 2014). Therefore, the VEGF-VEGFR2 signaling pathway has been extensively explored as a target for tumor therapy. To enhance cellular uptake and antiangiogenic activities in vitro and in vivo, $\mathrm{Fu}$ and colleagues conjugated RGD peptide to selenium nanoparticles loaded with doxorubicin (Dox) for targeting tumor vasculature. This nanosystem disassembled under acidic condition in lysosomes after internalization, triggering drug release. In vivo experiments showed inhibition of MCF-7 tumor growth and angiogenesis. The antiangiogenesis effect resulted from apoptosis and cell-cycle arrest in endothelial cells through downregulation of the VEGF-VEGFR2 signaling pathway (Fu et al., 2016). Inhibition of VEGFR2 mRNA expression in new tumor blood vessels is also an attractive approach for antitumor therapy. For tumor-targeted VEGFR2-siRNA delivery, Liu and colleagues (2014) designed a self-assembling peptide nanoparticle system consisting of the targeting cyclopeptide $\mathrm{c}$ (RGDfk) with an 8-amino-3,6dioxaoctanoic acid- $\beta$-maleimidopropionic acid modification (referred to as RPM) that is capable of encapsulating siRNA via intermolecular hydrogen bonding. The RPM/VEGFR2siRNA showed high gene- silencing efficiency and minimal cytotoxicity in vitro and effectively suppressed neovascularization when delivered into zebra fish embryos. Administration of RPM/VEGFR2-siRNA to tumor-bearing nude mice significantly inhibited tumor growth and reduced the density of tumor vessels, accompanied by downregulated VEGFR2, at both the mRNA and protein levels in tumor tissues. No measurable immunogenicity of the nanoparticles was observed in mice (Liu et al., 2014).

In addition to RGD peptide derivatives, many other peptides can target tumor blood vessels. For example, Herringson and coworkers (2011) tested two targeting peptides, peptide WHSDMEWWYLLG, an antagonist for VEGFR-1, and peptide ATWLPPR, which specifically binds to neuropilin-1, a VEGFR-2 coreceptor. When engrafted into Dox-containing liposomes, the peptide WHSDMEWWYLLG promoted liposome aggregation and/or leakage of the encapsulated liposomal drug. ATWLPPR liposomes showed significantly enhanced tumor targeting efficiency, particularly when 
PE-PEG750 was used as the stabilizing lipid instead of PE-PEG2000 in the construction of the liposomes. Moreover, ATWLPPR liposomes inhibited the growth of B16-F1 tumors in mice (Herringson and Altin, 2011) (Table 5).

Targeting Tumor Lymphatics and TAMs. Lymphatic vessels in tumors are morphologically distinct from normal lymphatic vessels. As shown in Table 1, LyP-1 is a ligand peptide that targets tumor cells, tumor lymphatics, and TAMs in tumor hypoxic regions (Sánchez-Martin et al., 2011). To improve the efficiency of targeted therapy of lymphatic metastatic tumors, Yan and colleagues (2012) developed LyP1-decorated and Dox-loaded PEGylated liposomes. Internalization of the LyP-1-decorated liposome was observed in tumor cells and metastatic lymph nodes, but not in normal lymph nodes. Peptide LyP-1 modification caused the liposomes to distribute adjacent to tumor lymphatics and TAMs in metastatic lymph nodes and also enhanced growth inhibition of tumor cells in vitro and lymphatic metastatic tumors in vivo (Yan et al., 2012).

TAMs are the predominant inflammatory cell components in the tumor microenvironment. Most polarize to become M2like macrophages, which as immunosuppressive populations contribute to angiogenesis and tumor immune evasion and subsequently promote tumor proliferation and metastasis. TAMs have also been reported to be the major players in chemoresistance and radioprotection in tumors (Jinushi and Komohara, 2015). In clinical studies, high densities and activation levels of TAMs are associated with poor treatment outcomes. Therefore, TAMs have become appealing targets for the development of cancer therapy. The most common strategies in TAM-targeted therapy include those that aim to inhibit macrophage recruitment, reduce TAM survival, enhance the M1-like tumoricidal activity of TAMs, or block their M2-like pro-tumor activity. Conde and colleagues (2015) designed nanoformulations composed of an RNA interference (RNAi)-peptide hybrid that specifically targets murine lung TAMs using M2pep peptide and delivered anti-VEGF siRNA into TAMs as well as tumor cells simultaneously. This approach achieved high targeting efficiency via M2pep and promoted effective VEGF downregulation. Administration of the hybrid nanoparticles resulted in immunomodulation of the TAM population within the tumor microenvironment and efficient eradication of tumors with an increased overall survival (Conde et al., 2015) (Table 5).

\section{Enhanced Tumor Penetration via Tumor Microenvironment Responsive Peptides}

Based on the pathophysiological variations in tumor microenvironment, many on-demand responsive nanoformulations have been designed for the spatially and temporally controlled release of therapeutic agents in response to specific stimuli. Some excellent review articles have already extensively discussed the molecular design and development of nanoscale systems for tumor microenvironment response (Mura et al., 2013; Wang et al., 2014). In this section, we focus on the peptides that are responsive to the tumor microenvironment as tools to enhance the ability of nanoformulations to penetrate tumors.

CPPs have received tremendous attention for their abilities to improve drug penetration in vivo as a result of their capability to mediate the internalization of a variety of cargo 
molecules into cells (Fonseca et al., 2009); however, the use of CPPs is limited in vivo owing to cell damage and low tissue specificity and to their positively charged nature (Zorko and Langel, 2005). Great efforts have been made to improve the selectivity of CPPs. Generally, CPPs are suppressed via electrostatically interacted shields or steric hindrance under physiologic conditions and are exposed to cells when they are circulating into tumor tissues. Based on this strategy, many nanosystems have been designed, including: 1) recovery of $\mathrm{CPP}$ functions via $\mathrm{pH}$-activatable systems, 2) recovery of $\mathrm{CPP}$ functions via protease-activatable systems, and 3) a combination of these two strategies (Huang et al., 2013c).

pH-Activatable Tumor Penetration. Owing to the excess metabolic acid secreted from fast-growing tumors, the $\mathrm{pH}$ in tumor microenvironment is more acidic than that in normal tissue, which makes the design of systems responsive to low $\mathrm{pH}$ an effective tumor-targeting strategy. Several pH-responsive systems have been developed that use the amino acid histidine, which has a pKa value of 6.5 and can be protonated from a net negative charge to a net positive charge when translocated into the acidic tumor microenvironment. Zhang and coworkers (2011) designed a pH-responsive cellpenetrating carrier based on histidine-rich peptide via a pH-triggered charge conversion strategy. Yeh and colleagues reported another $\mathrm{pH}$-responsive $\mathrm{CPP}$, in which $\mathrm{pH}$ sensitivity was controlled by recombinant fusion to a histidine-glutamine oligopeptide (Yeh et al., 2016). Zhao and coworkers (2016) found that polyhistidine could respond to the acidic tumor microenvironment by exposing a CPP R4 sequence. In the literature mentioned herein, cell internalization of nanocarriers can be promoted by means of $\mathrm{pH}$-triggered CPP surfacecharge reversal from negative to neutral or positive.

CPPS that are $\mathrm{pH}-$ responsive can facilitate the active targeting and uptake of tethered nanoparticles. Shi and colleagues (2015) designed a novel peptide containing a targeting peptide $\mathrm{c}(\mathrm{RGDfK})$ and a $\mathrm{pH}$-responsive CPP (AGYLLGHINLHHLAHL(Aib)HHIL-NH2). Nanoparticles conjugated with this RGD-CPP peptide were not only able to actively target $\alpha_{\nu} \beta_{3}$-overexpressing cells, compared with CPP-nanoparticles, but they also increased cellular uptake compared with RGD-nanoparticles. RGD-CPP-nanoparticles loaded with paclitaxel showed significantly higher survival rates in B16F10 tumor-bearing mice compared with other groups (Shi et al., 2015).

Enzyme-Activatable Tumor Penetration. Expression of MMPs is often upregulated in tumor ECM, and MMPs can cleave specific sequences via enzymatic catalysis. Thus, MMPs may serve as a better target for broad-spectrum theranostic applications. Nanoparticles incorporated with CPPs can be modified with PEG via an MMP-2 cleavable spacer to mask positively charged CPPs. Linkage with PEG was able to enhance the stability of nanoparticles in serum and avoid nonspecific interactions (Harada et al., 2010). For example, one group of investigators developed a liposome modified with CPP and a PEG-conjugated antibody. PEG was linked to the liposome via a MMP-2 responsive sequence (Zhu et al., 2012). Through the enhanced permeability and retention effect, these liposomes could selectively accumulate at the xenograft tumor site, where PEG was subsequently detached as a result of cleavage of the linker by endogenous MMP-2. In this manner, CPP-mediated cell penetration of the liposomes was achieved.
Wang and coworkers developed a tumor specific drugdelivery system by encapsulating nanovesicles with an MMPresponsive polymeric network. This polymeric network cover could effectively prevent the cargo from nonspecifically leaking from the nanoparticles, with enhanced drug bioavailability. Tumor-overexpressed MMP-2 specifically triggered the disassembly of polymeric networks and released the functional nanoparticles in tumor tissues. By using a tumor-homing peptide iRGD conjugated to the nanoparticles as a target ligand, they were able to facilitate tumor targeting and penetration of the drug both in vitro and in vivo. These results demonstrate that synergistic therapeutic efficacy can be achieved based on improved tumor accumulation, penetration, and MMP-responsive drug release in the tumor microenvironment (Liu et al., 2015).

pH and Enzyme Double-Activatable Tumor Penetration. Another strategy reported for tumor microenvironmentspecific recovery of the $\mathrm{CPP}$ effect is based on $\mathrm{pH}$ and protease double-activatable systems. Polyanionic peptides with $\mathrm{pH}$ responsive properties are used to neutralize the CPP charge. In the tumor microenvironment the charges of these masking peptides is altered to result in CPP dissociation. For example, Jiang's group devised a pH-sensitive masking peptide with an isoelectric point of approximately 6.4 (Huang et al., $2013 \mathrm{a}, \mathrm{b})$. This peptide is negatively charged under physiologic $\mathrm{pH}$, but it becomes predominantly uncharged or positively charged in the acidic tumor microenvironment. Since the conjugation of this masking peptide with CPP is weak, a peptide linker that is MMPs-cleavable was inserted between the masking peptide and CPP to form an intramolecular hairpin structure. When administrated in vivo, the hairpin structure remains intact in the circulation with the CPP blocked, maintaining the delivery system cell-impermeable and pharmacologically inactive in plasma. In the tumor microenvironment, the enzyme-responsive linker is cleaved by MMPs, and the hairpin structure disassociates. The exposed CPP can then penetrate the tumor. Based on this strategy, Jiang's group developed a series of tumor-targeting nanosystems with enhanced drug delivery and antitumor efficacy (Huang et al., 2013a,b).

\section{Peptide Self-Assembled Nanoformulations Targeting CAFs to Break Stromal Barriers}

Despite the promising therapeutic potential exhibited by numerous antitumor nanoformulations, the heterogeneity among tumor cells and the presence of complex stromal cell barriers still present great challenges that limit tumortargeting and cell-penetrating performance. Therefore, strategies to overcome tumor heterogeneity and to break stromal barriers are urgently needed. CAFs, the major stromal cell type in tumor microenvironment, play a key role in the formation of stromal barriers, leading to poor penetration for particulate therapeutics and also limiting access of pharmacologic drugs. Nanoformulations specifically targeting CAFs have shown promising results, as discussed in the following sections.

Our group developed peptide-based nanoformulations targeting and depleting CAFs to overcome the aforementioned obstacles. For example, a dual-mode nanomaterial that used CAF targeting combined with increased cellular uptake 

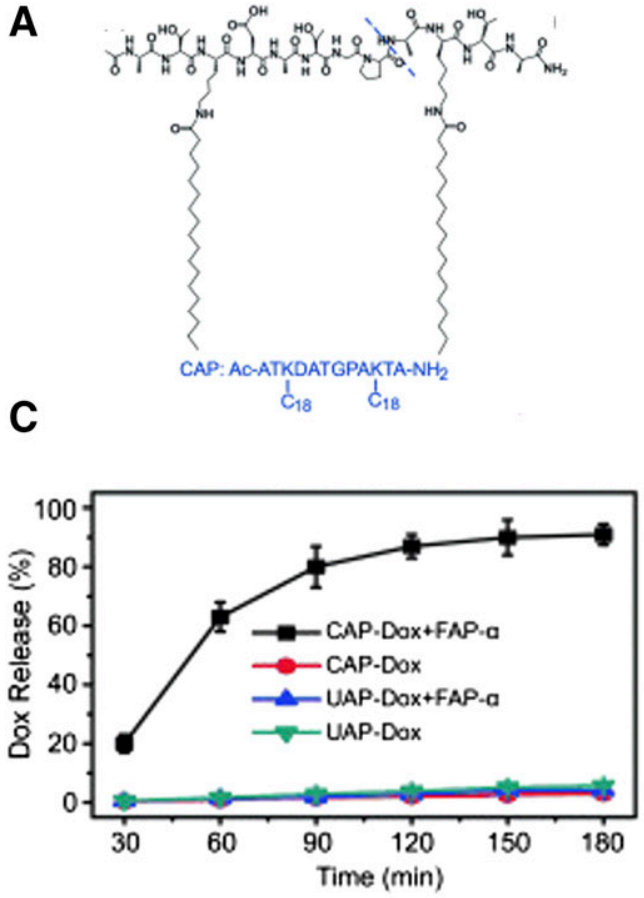
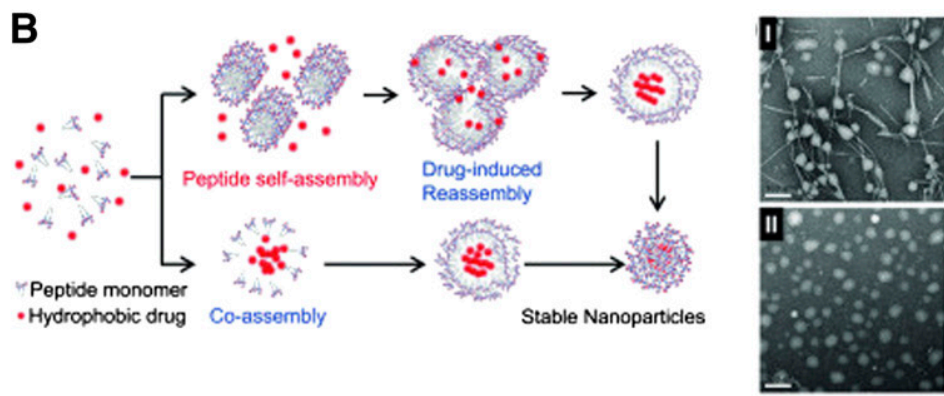

D
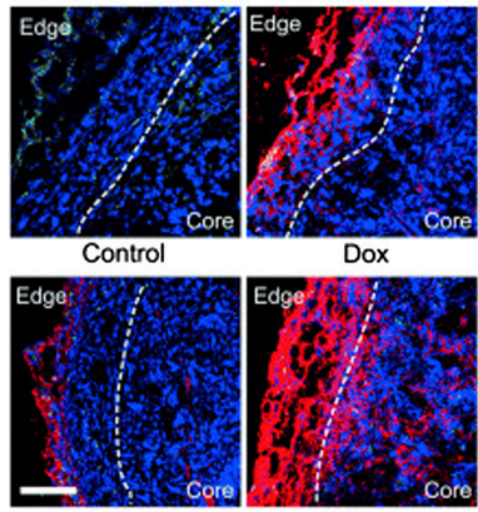

UAP-Dox

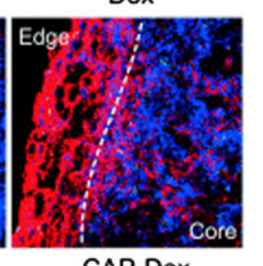

E

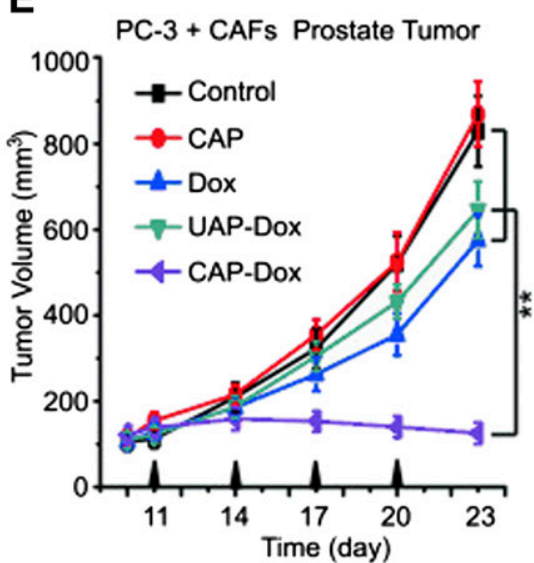

Fig. 1. FAP- $\alpha$ responsive nanocarriers based on a cleavable amphiphilic peptide. (A) The structure of peptide cleavable amphiphilic peptide (CAP), which contains a TGPA sequence that can be cleaved by FAP- $\alpha$. (B) Proposed mechanism of peptide self-assembly, drug-induced reassembly, and peptide and drug coassembly in the hydrophobic drug and amphiphilic peptide mixed solution. The components form the stable nanoparticles. The morphology of peptide assembly during Dox loading was observed by transmission electron microscopy. The assemblies transformed from mace-like (I) to spherical (II) with prolonged ultrasonication. (C) Drug-release profiles of CAP-Dox and uncleavable amphiphilic peptide (UAP)-Dox) in the presence or absence of FAP$\alpha$. (D) Penetration of Dox into prostate tumor (PC-3 and CAF coimplanted) tissues after intravenous injection of different Dox formulations. Frozen tumor sections were stained with DAPI (blue) to label nuclei and CD31 (green) antibody to label tumor vasculature. Red: Dox. (E) Growth curves of PC-3 and CAF coimplanted prostate tumors in mice treated with different Dox formulations (used with permission from Angew Chem Int Ed, John Wiley \& Sons; Ji et al., 2016b.). $* * P<0.01$ vs. Control, Dox, and UAP-Dox groups.

coordinated by CPP and cholesterol improved the tumor penetration of chemotherapeutic drugs. This was achieved by depletion of CAFs and disruption of stromal barriers for the treatment of CAF-rich solid tumors (Ji et al., 2015). In another study, we reported a novel CAF-targeting drug delivery nanosystem based on a cleavable amphiphilic peptide designed to be specifically responsive to FAP- $\alpha$, a membranebound serine protease specifically expressed on CAFs (Ji et al., 2016b). This cleavable amphiphilic peptide nanocarrier transformed from self-assembled nanofibers to spherical nanoparticles when loaded with hydrophobic drugs. The disassembly of these nanoparticles upon FAP- $\alpha$ cleavage resulted in efficient release of the encapsulated drugs specifically at tumor sites. This "transformer"-like drug nanocarrier could also disrupt the stromal barrier and enhance local drug accumulation (Fig. 1).

Since CAFs encompass a multifunctional stromal cell type, we found that selective inhibition, rather than complete depletion of CAFs, may be more appropriate for a safer longterm effective strategy. In one study, we developed a $\beta$-cyclodextrin ( $\beta$-CD) modified MMP-2-responsive liposome loaded with the antifibrotic and anti-inflammatory agent pirfenidone and the chemotherapeutic drug gemcitabine (GEM) for CAF regulation; this formulation was used for targeted delivery of GEM in pancreatic cancer therapy (Ji et al., 2016a). When this nanoformulation reached the tumor site, the pirfenidoneloaded $\beta$-CD was able to discharge and accumulate in the stroma after cleavage of the MMP-2 substrate peptide. The initial release of pirfenidone effectively downregulated fibrosis and decreased the stromal barrier. Subsequently, the RGD-containing liposomes loaded with GEM recognized tumor cells and penetrated into the tumor tissue, resulting in enhanced therapeutic efficacy. This combined antifibrosis and antitumor strategy may increase drug penetration and enhance chemotherapeutic efficacy, providing a potential strategy for the design of nanoformulations to improve the pancreatic tumor therapy (Fig. 2).

\section{Therapeutic Peptide Self-Assembled Nanoformulations Targeting Tumor Vasculatures}

The short circulating half-life of small therapeutic peptides in vivo may limit their use in human clinical applications for tumor therapy (Talmadge, 1998). Strategies are needed for increasing the stability and activity of peptide drugs to improve their therapeutic outcomes. Apart from chemical modification, incorporating therapeutic peptides into nanosystems with adequate size, morphology, and surface properties can improve their stability and pharmacokinetics. Mediated by intermolecular forces, amphiphilic peptides composed of distinct hydrophobic and hydrophilic segments are able to self-assemble into particular nanostructures. A therapeutic peptide with the ability to inhibit CXC chemokine 
A

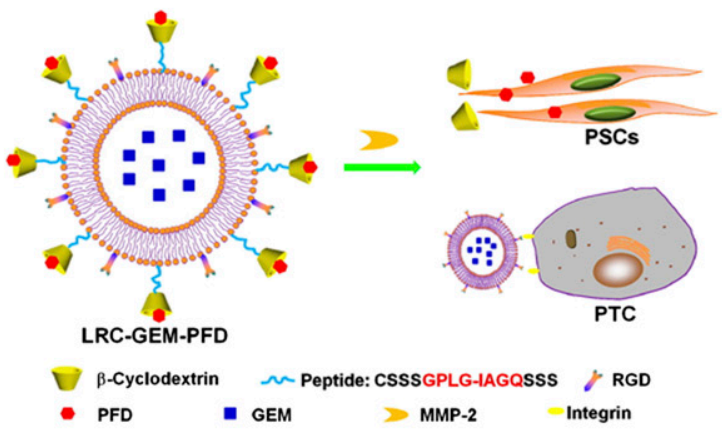

C

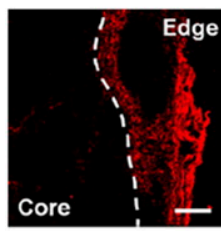

Control

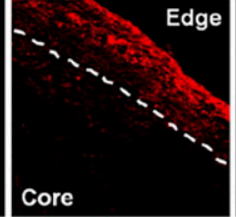

LRC

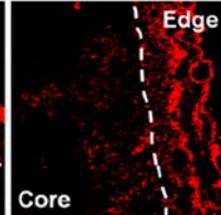

PFD

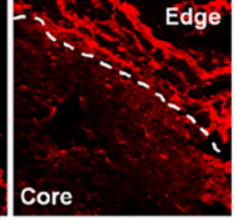

LRC-PFD
B

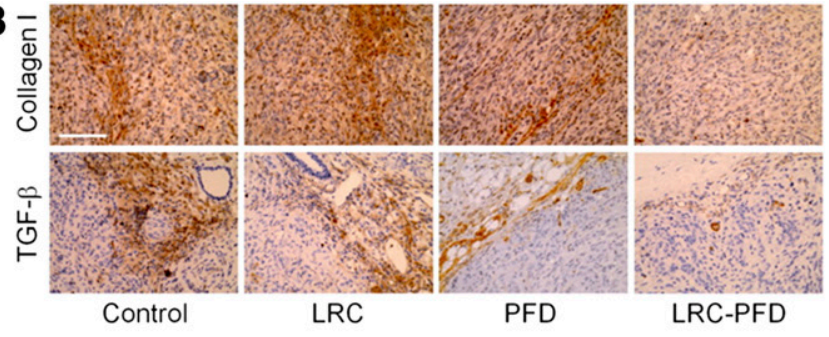

D

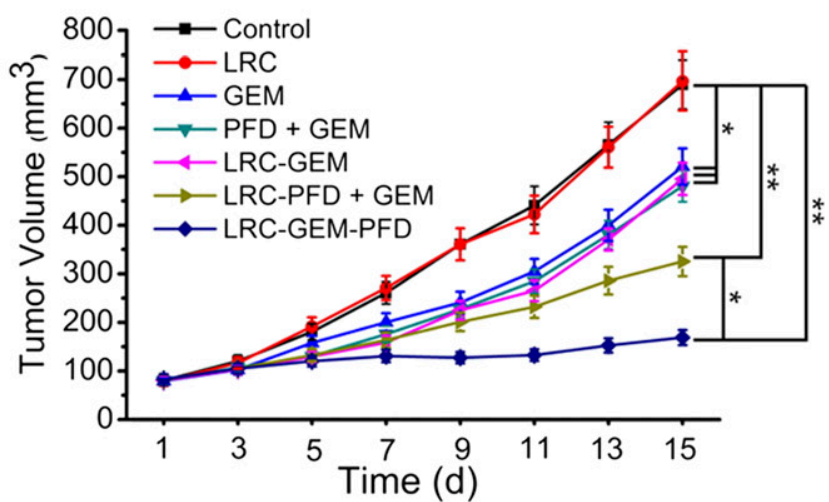

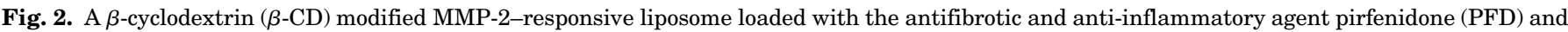

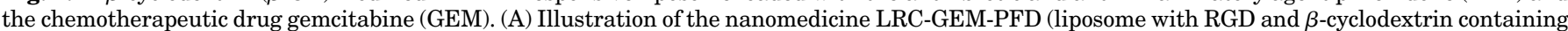

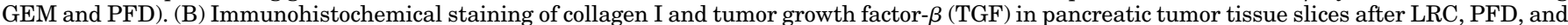

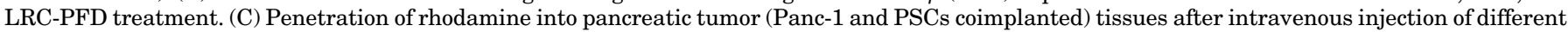

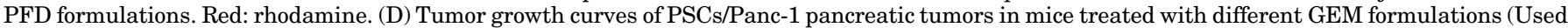
with permission from ACS Appl Mater Interfaces, American Chemical Society; Ji et al., 2016a.). ${ }^{*} P<0.05$, ${ }^{*} P<<0.01$ vs. Control.

receptor 4 function was demonstrated to form self-assembled nanoparticles that hindered CXC chemokine receptor 4-mediated tumor metastasis (Tarasov et al., 2011).

Inspired by the peptide self-assembly concept, we designed a tumor microenvironment-responsive nanoformulation based on a tailor-designed amphiphilic peptide through conjugation of functional 3-diethylaminopropylisothiocyanate (DEAP) molecules to a short peptide (Zhao et al., 2014). The apparent $\mathrm{pK}_{\mathrm{b}}$ of peptide conjugated with DEAP was approximately 6.9 , which is close to the $\mathrm{pH}$ range within the tumor microenvironment. Protonation of the amphiphilic peptide at a weakly acidic $\mathrm{pH}$ led to a reduction in hydrophobicity, which triggered a $\mathrm{pH}$-induced "intelligent switch" for the peptide self-assembled nanostructures, subsequently leading to their disassembly in the tumor microenvironment (Zhao et al., 2014). Furthermore, we recently developed a smart self-assembled nanoformulation using a therapeutic peptide known as C16Y (DFKLFAVYIKYR). This peptide, with a hydrophilic head and DEAP as a hydrophobic tail, inhibits angiogenesis and tumor growth by targeting integrin $\alpha_{\mathrm{V}} \beta_{3}$ and $\alpha_{\mathrm{V}} \beta_{1}$ (Ding et al., 2015). DEAP-C16Y peptides selfassembled into spherical nanostructures under physiologic conditions and subsequently dissociated to release individual peptide molecules in weakly acidic tumors. Mechanistic investigation revealed that dissociated DEAP-C16Y peptides inhibited vascular endothelial cell migration and tubule formation through suppression of the focal adhesion kinase and PI3K-Akt signaling axis and also restrained tumor cell invasion via downregulation of invadopodia formation. The systemically administered DEAPC16Y nanoformulations significantly decreased microvessel density, tumor growth, and distant metastasis formation in nude mice, with effectively prolonged blood circulation of the therapeutic peptide in vivo. Additionally, the DEAP-C16Y nanostructures can be a smart and effective drug-delivery system of antineoplastic agents for targeted combination therapy. In this study, for the first time, we developed a selfassembled nanoformulation by using a therapeutic peptide as a building block. This peptide showed intrinsic antitumor activity and also provided a platform for combination therapy by encapsulating chemotherapeutic drugs in the nanoformulation, demonstrating a potential strategy for the future design of antitumor nanotherapeutics (Fig. 3) (Ding et al., 2015).

Numerous tumor microenvironment targeted nanosystems have been constructed and optimized using functional peptides to mediate cell penetration, target the tumor microenvironment, respond to tumor-specific enzymes for controlled drug release, or regulate cellular signaling transduction pathways. According to their functionalities, building blocks in nanoformulations can be classified into different categories and regarded as modules for nanosystem construction. For more efficient design and implementation of peptide-based drug delivery, controlled release, and targeting, as well as the regulation of the tumor microenvironment, we propose a modularized concept for peptide nanosystem construction (Fig. 4A). The self-assembled micelles, vesicles, and nanofibers are assembled from amphipathic monomers in a process that is generally mediated by various intermolecular forces. Typically, functional peptides are used as the hydrophilic heads, and lipophilic molecules such as cholesterol, alkyl chains, polymers, and lipids serve as the hydrophobic tails. 

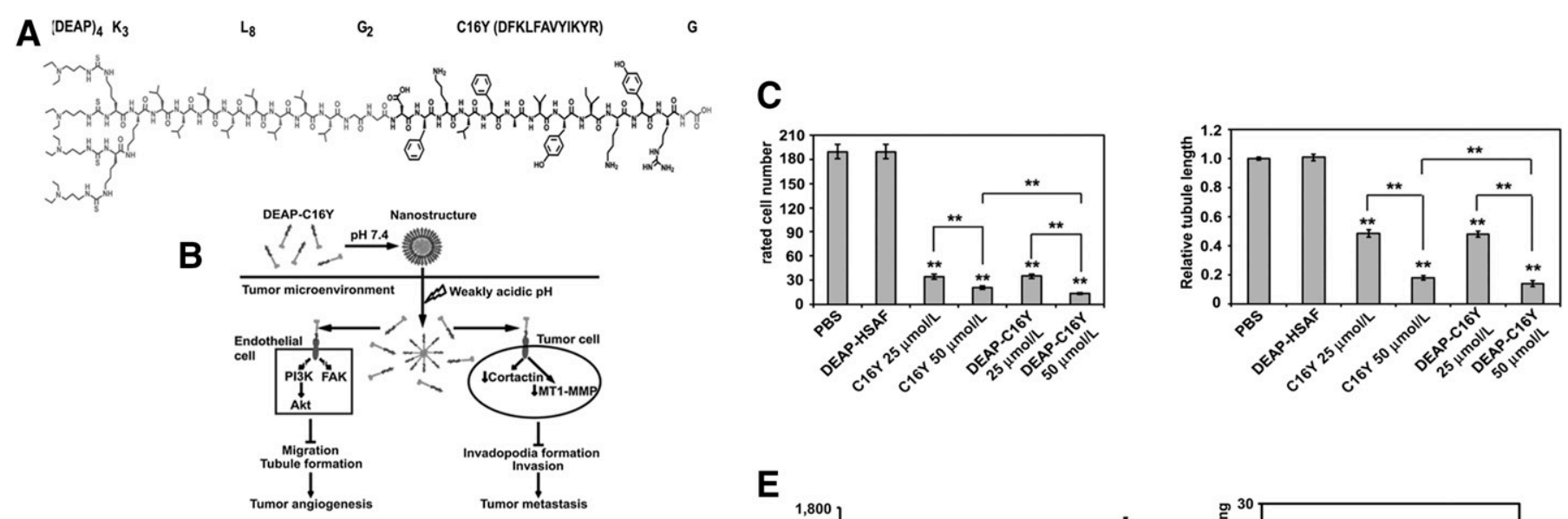

D
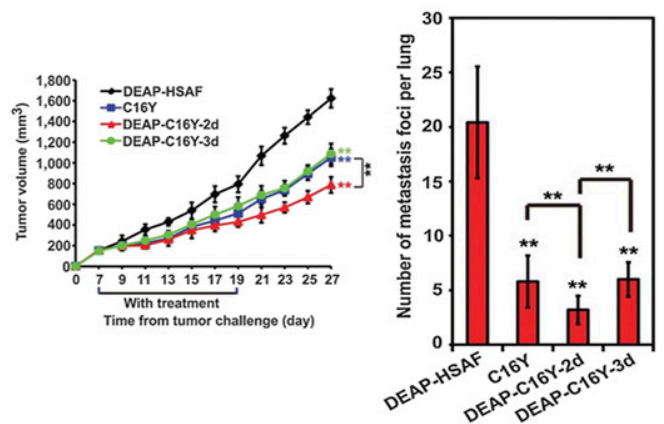

E

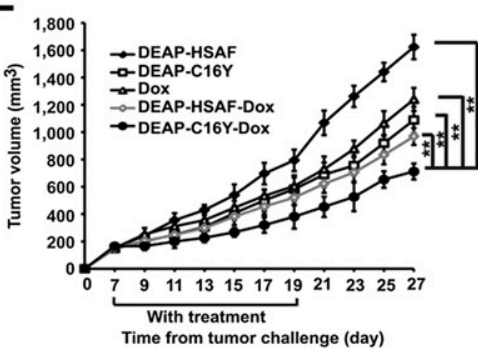

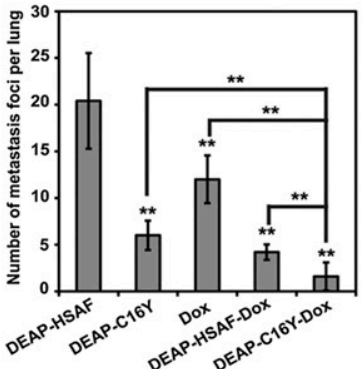

Fig. 3. A smart therapeutic peptide for inhibiting angiogenesis and tumor growth. (A) Schematic structure of the DEAP-C16Y peptide. (B) Proposed antitumor mechanism of DEAP-C16Y nanostructures. (C) Migration of human umbilical vein endothelial cell (HUVECs) treated by peptides in a transwell migration assay; cells were chemoattracted by 5\% fetal bovine serum/RPMI-1640 medium. The number of migrated cells in each group was quantified. Tubule formation was assessed in HUVECs treated by C16Y or DEAP-C16Y. The total network length in each treatment group was quantified. $* P<0.05, * * P<0.01$ compared with the PBS group. (D) Growth curves and metastatic foci number of 4T1 tumors. Mice bearing 4T1 tumors were treated with DEAP-HSAF: a fragment of human serum albumin, DELRDEGKASSAKQ. nanostructures (negative control) or with C16Y or DEAP$\mathrm{C} 16 \mathrm{Y}$ nanostructures for indicated periods. Tumor volume was calculated every other day. Lung sections from 4T1-bearing mice were stained with H\&E or proliferating cell nuclear antigens. The number of metastatic foci was quantified. $* P<0.05, * * P<0.01$ compared with DEAP-HSAF group. (E) Antitumor efficacy of DEAP-C16Y-Dox nanostructures. Mice bearing 4T1 tumors were treated with the indicated formulations every third day. Tumor volume was calculated every other day. The number of metastatic foci per lung was quantified (Used with permission from Mol Cancer Ther, American Association for Cancer Research; Ding et al., 2015). *P<0.05, $* * P<0.01$ compared with DEAP-HSAF group.

Such monomers, constructed with multiple modules, then selfassemble into nanostructures with diverse functions and physicochemical features. Apart from this self-assembled system, there is another common type of peptide-based nanosystem that uses preformed nanostructures, such as liposomes, polymeric particles, or inorganic nanostructures (e.g., gold, silicon, and ferric oxide) onto surfaces to achieve improved biocompatibility, active targeting, controlled release, or enhanced tissue penetration (Fig. 4B).

By proposing the concept of modularized construction, our intention is to highlight a strategy that categorizes the building blocks into different modules, depending on their functions. The desirable modules suitable for targeting environment and encapsulated therapeutic molecules can be selected and then combined to construct intelligent and well controlled nanoformulations. Modularized construction makes it easier to construct nanoformulations with promising specificity and therapeutic efficacy.

\section{Conclusions}

Compared with tumor cells, the surrounding tumor microenvironment is a more accessible target for antitumor therapy. The specifically expressed receptors and unique physicochemical conditions within the tumor microenvironment provide many opportunities for nanoformulations to target tumor tissues. Many nanosystems have been developed to target the tumor microenvironment with the goal of achieving enhanced targeting efficacy and therapeutic outcomes. As discussed in this review, tumor progression is promoted by the

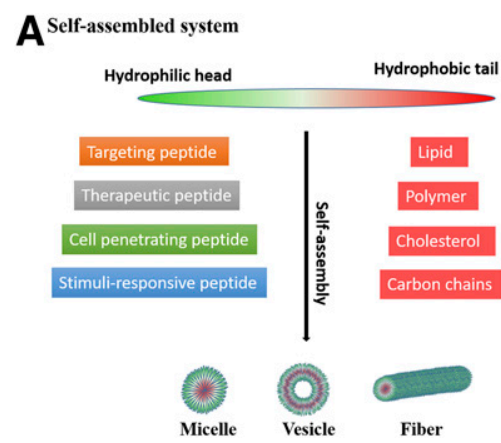

B Modified system

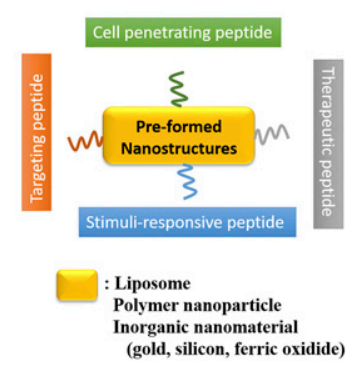

Fig. 4. Modularized construction of nanoformulations. (A) Amphipathic peptide consisting of a hydrophilic head and hydrophobic tail, based on various modules that self assembles into nanostructures such as micelles, vesicles, and fibers in response to intermolecular forces. (B) Preformed nanostructures can be modified with functional modules for enhanced therapeutic efficacy. 
sustained influence of the tumor microenvironment. In addition, stromal cells and ECM in the tumor microenvironment generate a natural sanctuary for tumor cells. Therapies that regulate the components in the tumor microenvironment, such as tumor blood vessels and CAFs, have been reported to efficiently suppress tumor growth with reduction of several underlying threats, such as invasion and metastasis. Moreover, the novel therapeutic strategy based on the dual targeting of tumor microenvironment and tumor cells has become increasingly attractive and has exhibited increased drug accessibility and elevated treatment efficacy. In particular, incorporated functional peptides are able to endow antitumor nanoformulations with high specificity, cell-penetrating ability, sensitivity to slight change in conditions, and potential for cellular pathway regulation and have achieved excellent therapeutic efficacy. In this review, we have proposed a modularized construction strategy for the assembly of peptide-based nanoformulations and have discussed its potential advantages and utility in anticancer nanosystem development. It should be noted that, despite the great advances in tumor microenvironment targeting and therapy, additional functional peptides need to be designed. A deeper understanding of the tumor microenvironment can also help us to make full use of its properties to develop antitumor nanoformulations with better specificity and stronger therapeutic efficacy.

\section{Authorship Contributions}

Wrote or contributed to the writing of the manuscript: Qin, Ding, Mujeeb, Zhao, Nie.

\section{References}

Al-Ahmady ZS, Al-Jamal WT, Bossche JV, Bui TT, Drake AF, Mason AJ, and Kostarelos K (2012) Lipid-peptide vesicle nanoscale hybrids for triggered drug release by mild hyperthermia in vitro and in vivo. ACS Nano 6:9335-9346.

Amand HL, Fant K, Nordén B, and Esbjörner EK (2008) Stimulated endocytosis in penetratin uptake: effect of arginine and lysine. Biochem Biophys Res Commun 371:621-625.

An M, Wijesinghe D, Andreev OA, Reshetnyak YK, and Engelman DM (2010) pH(low)-insertion-peptide (pHLIP) translocation of membrane impermeable phalloidin toxin inhibits cancer cell proliferation. Proc Natl Acad Sci USA 107 20246-20250.

Andreev OA, Dupuy AD, Segala M, Sandugu S, Serra DA, Chichester CO, Engelman DM, and Reshetnyak YK (2007) Mechanism and uses of a membrane peptide that targets tumors and other acidic tissues in vivo. Proc Natl Acad Sci USA 104: $7893-7898$.

Arap W, Pasqualini R, and Ruoslahti E (1998) Cancer treatment by targeted drug delivery to tumor vasculature in a mouse model. Science 279:377-380.

Carmeliet P (2000) Mechanisms of angiogenesis and arteriogenesis. Nat Med 6: 389-395

Chang HN, Liu BY, Qi YK, Zhou Y, Chen YP, Pan KM, Li WW, Zhou XM, Ma WW, Fu CY, et al. (2015) Blocking of the PD-1/PD-L1 interaction by a D-peptide antagonist for cancer immunotherapy. Angew Chem Int Ed Engl 54:11760-11764.

Cieslewicz M, Tang J, Yu JL, Cao H, Zavaljevski M, Motoyama K, Lieber A, Raines EW, and Pun SH (2013) Targeted delivery of proapoptotic peptides to tumorassociated macrophages improves survival. Proc Natl Acad Sci USA 110: 15919-15924.

Conde J, Bao C, Tan Y, Cui D, Edelman ER, Azevedo HS, Byrne HJ, Artzi N, and Tian F (2015) Dual targeted immunotherapy via in vivo delivery of biohybrid RNAi-peptide nanoparticles to tumour-associated macrophages and cancer cells. Adv Funct Mater 25:4183-4194.

Danhier F, Feron O, and Préat V (2010) To exploit the tumor microenvironment: passive and active tumor targeting of nanocarriers for anti-cancer drug delivery. $J$ Control Release 148:135-146.

Danhier F, Le Breton A, and Préat V (2012) RGD-based strategies to target alpha(v) beta(3) integrin in cancer therapy and diagnosis. Mol Pharm 9:2961-2973.

Daquinag AC, Zhang Y, Amaya-Manzanares F, Simmons PJ, and Kolonin MG (2011) An isoform of decorin is a resistin receptor on the surface of adipose progenitor cells. Cell Stem Cell 9:74-86.

Derossi D, Joliot AH, Chassaing G, and Prochiantz A (1994) The third helix of the Antennapedia homeodomain translocates through biological membranes. J Biol Chem 269:10444-10450.

Ding Y, Ji T, Zhao Y, Zhang Y, Zhao X, Zhao R, Lang J, Zhao X, Shi J, Sukumar S, et al. (2015) Improvement of stability and efficacy of C16Y therapeutic peptide via molecular self-assembly into tumor-responsive nanoformulation. Mol Cancer Ther 14:2390-2400
Dokka S, Toledo-Velasquez D, Shi X, Wang L, and Rojanasakul Y (1997) Cellular delivery of oligonucleotides by synthetic import peptide carrier. Pharm Res 14 1759-1764.

El-Andaloussi S, Johansson HJ, Holm T, and Langel U (2007) A novel cellpenetrating peptide, M918, for efficient delivery of proteins and peptide nucleic acids. Mol Ther 15:1820-1826.

Elliott G and O'Hare P (1997) Intercellular trafficking and protein delivery by a herpesvirus structural protein. Cell 88:223-233.

Elmquist A, Hansen M, and Langel U (2006) Structure-activity relationship study of the cell-penetrating peptide pVEC. Biochim Biophys Acta 1758:721-729.

Fernández-Carneado J, Kogan MJ, Castel S, and Giralt E (2004) Potential peptide carriers: amphipathic proline-rich peptides derived from the N-terminal domain of gamma-zein. Angew Chem Int Ed Engl 43:1811-1814.

Fogal V, Zhang L, Krajewski S, and Ruoslahti E (2008) Mitochondrial/cell-surface protein $\mathrm{p} 32 / \mathrm{gC} 1 \mathrm{qR}$ as a molecular target in tumor cells and tumor stroma. Cancer Res 68:7210-7218.

Fonseca SB, Pereira MP, and Kelley SO (2009) Recent advances in the use of cellpenetrating peptides for medical and biological applications. Adv Drug Deliv Rev 61:953-964.

Fu X, Yang Y, Li X, Lai H, Huang Y, He L, Zheng W, and Chen T (2016) RGD peptideconjugated selenium nanoparticles: antiangiogenesis by suppressing VEGFVEGFR2-ERK/AKT pathway. Nanomedicine (Lond) 12:1627-1639.

Gao W, Xiang B, Meng TT, Liu F, and Qi XR (2013) Chemotherapeutic drug delivery to cancer cells using a combination of folate targeting and tumor microenvironment-sensitive polypeptides. Biomaterials 34:4137-4149.

Gautam A, Kapoor P, Chaudhary K, Kumar R, and Raghava GPS (2014) Tumor homing peptides as molecular probes for cancer therapeutics, diagnostics and theranostics. Curr Med Chem 21:2367-2391.

Gvozdenovic A, Boro A, Meier D, Bode-Lesniewska B, Born W, Muff R, and Fuchs B (2016) Targeting $\alpha v \beta 3$ and $\alpha v \beta 5$ integrins inhibits pulmonary metastasis in an intratibial xenograft osteosarcoma mouse model. Oncotarget 7:55141-55154.

Harada A, Kimura Y, Kojima C, and Kono K (2010) Effective tolerance to serum proteins of head-tail type polycation vectors by PEGylation at the periphery of the head block. Biomacromolecules 11:1036-1042.

Hariton-Gazal E, Feder R, Mor A, Graessmann A, Brack-Werner R, Jans D, Gilon C, and Loyter A (2002) Targeting of nonkaryophilic cell-permeable peptides into the nuclei of intact cells by covalently attached nuclear localization signals. Biochemistry 41:9208-9214.

Hatakeyama S, Sugihara K, Shibata TK, Nakayama J, Akama TO, Tamura N, Wong SM, Bobkov AA, Takano Y, Ohyama C, et al. (2011) Targeted drug delivery to tumor vasculature by a carbohydrate mimetic peptide. Proc Natl Acad Sci USA 108:19587-19592.

Herringson TP and Altin JG (2011) Effective tumor targeting and enhanced antitumor effect of liposomes engrafted with peptides specific for tumor lymphatics and vasculature. Int $J$ Pharm 411:206-214.

Huang S, Shao K, Kuang Y, Liu Y, Li J, An S, Guo Y, Ma H, He X, and Jiang C (2013a) Tumor targeting and microenvironment-responsive nanoparticles for gene delivery. Biomaterials 34:5294-5302.

Huang S, Shao K, Liu Y, Kuang Y, Li J, An S, Guo Y, Ma H, and Jiang C (2013b) Tumor-targeting and microenvironment-responsive smart nanoparticles for combination therapy of antiangiogenesis and apoptosis. ACS Nano 7 $2860-2871$.

Huang Y, Jiang Y, Wang H, Wang J, Shin MC, Byun Y, He H, Liang Y, and Yang VC (2013c) Curb challenges of the "Trojan Horse" approach: smart strategies in achieving effective yet safe cell-penetrating peptide-based drug delivery. Adv Drug Deliv Rev 65:1299-1315.

Islam MZ, Ariyama H, Alam JM, and Yamazaki M (2014) Entry of cell-penetrating peptide transportan 10 into a single vesicle by translocating across lipid membrane and its induced pores. Biochemistry 53:386-396.

Ji T, Ding Y, Zhao Y, Wang J, Qin H, Liu X, Lang J, Zhao R, Zhang Y, Shi J, et al. (2015) Peptide assembly integration of fibroblast-targeting and cell-penetration features for enhanced antitumor drug delivery. Adv Mater 27:1865-1873.

Ji T, Li S, Zhang Y, Lang J, Ding Y, Zhao X, Zhao R, Li Y, Shi J, Ha, et al. (2016a) An MMP-2 responsive liposome integrating antifibrosis and chemotherapeutic drugs for enhanced drug perfusion and efficacy in pancreatic cancer. ACS Appl Mater Interfaces 8:3438-3445.

Ji T, Zhao Y, Ding Y, and Nie G (2013) Using functional nanomaterials to target and regulate the tumor microenvironment: diagnostic and therapeutic applications. Adv Mater 25:3508-3525.

Ji T, Zhao Y, Ding Y, Wang J, Zhao R, Lang J, Qin H, Liu X, Shi J, Tao N, et al. (2016b) Transformable peptide nanocarriers for expeditious drug release and ef fective cancer therapy via cancer-associated fibroblast activation. Angew Chem Int Ed Engl 55:1050-1055.

Jinushi M and Komohara Y (2015) Tumor-associated macrophages as an emerging target against tumors: creating a new path from bench to bedside. Biochim Biophys Acta 1855:123-130.

Jung H-K, Kim S, Park R-W, Park J-Y, Kim I-S, and Lee B (2016) Bladder tumortargeted delivery of pro-apoptotic peptide for cancer therapy. $J$ Control Release 235:259-267.

Khawar IA, Kim JH, and Kuh HJ (2015) Improving drug delivery to solid tumors: priming the tumor microenvironment. J Control Release 201:78-89.

Koivunen E, Arap W, Valtanen H, Rainisalo A, Medina OP, Heikkilä P, Kantor C, Gahmberg CG, Salo T, Konttinen YT, et al. (1999) Tumor targeting with a selective gelatinase inhibitor. Nat Biotechnol 17:768-774.

Laakkonen P, Porkka K, Hoffman JA, and Ruoslahti E (2002) A tumor-homing peptide with a targeting specificity related to lymphatic vessels. Nat Med 8 $751-755$.

Lee TY, Folkman J, and Javaherian K (2010) HSPG-binding peptide corresponding to the exon 6a-encoded domain of VEGF inhibits tumor growth by blocking angiogenesis in murine model. PLoS One 5:e9945. 
Liang X, Xu F, Li X, Ma C, Zhang Y, and Xu W (2014) VEGF signal system: the application of antiangiogenesis. Curr Med Chem 21:894-910.

Liu L, Liu X, Xu Q, Wu P, Zuo X, Zhang J, Deng H, Wu Z, and Ji A (2014) Selfassembled nanoparticles based on the c(RGDfk) peptide for the delivery of siRNA targeting the VEGFR2 gene for tumor therapy. Int J Nanomedicine 9:3509-3526.

Liu Y, Zhang D, Qiao ZY, Qi GB, Liang XJ, Chen XG, and Wang H (2015) A peptidenetwork weaved nanoplatform with tumor microenvironment responsiveness and deep tissue penetration capability for cancer therapy. Adv Mater 27:5034-5042.

Macewan SR and Chilkoti A (2012) Digital switching of local arginine density in a genetically encoded self-assembled polypeptide nanoparticle controls cellular uptake. Nano Lett 12:3322-3328.

Morris MC, Deshayes S, Heitz F, and Divita G (2008) Cell-penetrating peptides: from molecular mechanisms to therapeutics. Biol Cell 100:201-217.

Moshnikova A, Moshnikova V, Andreev OA, and Reshetnyak YK (2013) Antiproliferative effect of pHLIP-amanitin. Biochemistry 52:1171-1178.

Mura S, Nicolas J, and Couvreur P (2013) Stimuli-responsive nanocarriers for drug delivery. Nat Mater 12:991-1003.

Nakayama F, Yasuda T, Umeda S, Asada M, Imamura T, Meineke V, and Akashi M (2011) Fibroblast growth factor-12 (FGF12) translocation into intestinal epithelial cells is dependent on a novel cell-penetrating peptide domain: involvement of internalization in the in vivo role of exogenous FGF12. J Biol Chem 286 $25823-25834$.

Neri S, Hashimoto H, Kii H, Watanabe H, Masutomi K, Kuwata T, Date H, Tsuboi M, Goto K, Ochiai A, et al. (2016) Cancer cell invasion driven by extracellular matrix remodeling is dependent on the properties of cancer-associated fibroblasts. $J$ Cancer Res Clin Oncol 142:437-446.

Nobuoka D, Yoshikawa T, Takahashi M, Iwama T, Horie K, Shimomura M, Suzuki S, Sakemura N, Nakatsugawa M, Sadamori H, et al. (2013) Intratumoral peptide injection enhances tumor cell antigenicity recognized by cytotoxic $\mathrm{T}$ lymphocytes: a potential option for improvement in antigen-specific cancer immunotherapy. Cancer Immunol Immunother 62:639-652.

Pasqualini R, Koivunen E, and Ruoslahti E (1997) Alpha v integrins as receptors for tumor targeting by circulating ligands. Nat Biotechnol 15:542-546.

Pilch J, Brown DM, Komatsu M, Järvinen TA, Yang M, Peters D, Hoffman RM, and Ruoslahti E (2006) Peptides selected for binding to clotted plasma accumulate in tumor stroma and wounds. Proc Natl Acad Sci USA 103:2800-2804.

Ponce ML, Hibino S, Lebioda AM, Mochizuki M, Nomizu M, and Kleinman HK (2003) Identification of a potent peptide antagonist to an active laminin-1 sequence that blocks angiogenesis and tumor growth. Cancer Res 63:5060-5064.

Porkka K, Laakkonen P, Hoffman JA, Bernasconi M, and Ruoslahti E (2002) A fragment of the HMGN2 protein homes to the nuclei of tumor cells and tumor endothelial cells in vivo. Proc Natl Acad Sci USA 99:7444-7449.

Rogers TL and Holen I (2011) Tumour macrophages as potential targets of bisphosphonates. J Transl Med 9:177.

Rosca EV, Koskimaki JE, Rivera CG, Pandey NB, Tamiz AP, and Popel AS (2011) Anti-angiogenic peptides for cancer therapeutics. Curr Pharm Biotechnol 12 $1101-1116$.

Sánchez-Martín D, Cuesta AM, Fogal V, Ruoslahti E, and Alvarez-Vallina L (2011) The multicompartmental p32/gClqR as a new target for antibody-based tumor targeting strategies. J Biol Chem 286:5197-5203.

Shi K, Li J, Cao Z, Yang P, Qiu Y, Yang B, Wang Y, Long Y, Liu Y, Zhang Q, et al (2015) A pH-responsive cell-penetrating peptide-modified liposomes with active recognizing of integrin $\alpha \mathrm{v} \beta 3$ for the treatment of melanoma. J Control Release $\mathbf{2 1 7}$ $138-150$.

Simberg D, Duza T, Park JH, Essler M, Pilch J, Zhang L, Derfus AM, Yang M, Hoffman RM, Bhatia S, et al. (2007) Biomimetic amplification of nanoparticle homing to tumors. Proc Natl Acad Sci USA 104:932-936.

Sugahara KN, Teesalu T, Karmali PP, Kotamraju VR, Agemy L, Greenwald DR, and Ruoslahti E (2010) Coadministration of a tumor-penetrating peptide enhances the efficacy of cancer drugs. Science 328:1031-1035.
Takeshima K, Chikushi A, Lee KK, Yonehara S, and Matsuzaki K (2003) Translocation of analogues of the antimicrobial peptides magainin and buforin across human cell membranes. $J$ Biol Chem 278:1310-1315.

Talmadge JE (1998) Pharmacodynamic aspects of peptide administration biological response modifiers. Adv Drug Deliv Rev 33:241-252.

Tarasov SG, Gaponenko V, Howard OM, Chen Y, Oppenheim JJ, Dyba MA, Subramaniam S, Lee Y, Michejda C, and Tarasova NI (2011) Structural plasticity of a transmembrane peptide allows self-assembly into biologically active nanoparticles. Proc Natl Acad Sci USA 108:9798-9803.

Tournaire R, Simon MP, le Noble F, Eichmann A, England P, and Pouysségur J (2004) A short synthetic peptide inhibits signal transduction, migration and angiogenesis mediated by Tie2 receptor. EMBO Rep 5:262-267.

Trabulo S, Cardoso AL, Mano M, and De Lima MC (2010) Cell-penetrating peptides: mechanisms of cellular uptake and generation of delivery systems. Pharmaceuticals (Basel) 3:961-993.

Uchida M, Kosuge H, Terashima M, Willits DA, Liepold LO, Young MJ, McConnell MV, and Douglas T (2011) Protein cage nanoparticles bearing the LyP-1 peptide for enhanced imaging of macrophage-rich vascular lesions. ACS Nano 5: 2493-2502.

Walrant A, Correia I, Jiao CY, Lequin O, Bent EH, Goasdoué N, Lacombe C, Chassaing G, Sagan S, and Alves ID (2011) Different membrane behaviour and cellular uptake of three basic arginine-rich peptides. Biochim Biophys Acta 1808:382-393.

Wang J, Liu Y, Teesalu T, Sugahara KN, Kotamrajua VR, Adams JD, Ferguson BS, Gong Q, Oh SS, Csordas AT, et al. (2011) Selection of phage-displayed peptides on live adherent cells in microfluidic channels. Proc Natl Acad Sci USA 108: 6909-6914.

Wang Y, Shim MS, Levinson NS, Sung HW, and Xia Y (2014) Stimuli-responsive materials for controlled release of theranostic agents. Adv Funct Mater 24 4206-4220.

Yan Z, Wang F, Wen Z, Zhan C, Feng L, Liu Y, Wei X, Xie C, and Lu W (2012) LyP-1conjugated PEGylated liposomes: a carrier system for targeted therapy of lymphatic metastatic tumor. J Control Release 157:118-125.

Yeh TH, Chen YR, Chen SY, Shen WC, Ann DK, Zaro JL, and Shen LJ (2016) Selective intracellular delivery of recombinant arginine deiminase (ADI) using $\mathrm{pH}$-sensitive cell penetrating peptides to overcome ADI resistance in hypoxic breast cancer cells. Mol Pharm 13:262-271.

Zhang W, Song J, Zhang B, Liu L, Wang K, and Wang R (2011) Design of acidactivated cell penetrating peptide for delivery of active molecules into cancer cells. Bioconjug Chem 22:1410-1415.

Zhao Y, Ji T, Wang H, Li S, Zhao Y, and Nie G (2014) Self-assembled peptide nanoparticles as tumor microenvironment activatable probes for tumor targeting and imaging. $J$ Control Release 177:11-19.

Zhao Y, Ren W, Zhong T, Zhang S, Huang D, Guo Y, Yao X, Wang C, Zhang WQ, Zhang X, et al. (2016) Tumor-specific $\mathrm{pH}$-responsive peptide-modified $\mathrm{pH}$-sensitive liposomes containing doxorubicin for enhancing glioma targeting and anti-tumor activity. J Control Release 222:56-66.

Zhu L, Kate P, and Torchilin VP (2012) Matrix metalloprotease 2-responsive multifunctional liposomal nanocarrier for enhanced tumor targeting. ACS Nano 6: 3491-3498.

Zorko M and Langel U (2005) Cell-penetrating peptides: mechanism and kinetics of cargo delivery. Adv Drug Deliv Rev 57:529-545.

Address correspondence to: Prof. Guangjun Nie, CAS Key Laboratory for Biomedical Effects of Nanomaterials and Nanosafety \& CAS Center for Excellence in Nanoscience, National Center for Nanoscience and Technology, No. 11 Beiyitiao, Zhongguancun, Beijing 100190, China. E-mail: niegi@ nanoctr.cn or Prof. Ying Zhao, University of Chinese Academy of Sciences, Beijing 100049, China. E-mail: zhaoying@nanoctr.cn 